\title{
Anti-inflammatory potentials of incretin-based therapies used in the management of diabetes
}

Habib Yaribeygi ${ }^{1^{*}}$, Mina Maleki ${ }^{2}$, Thozhukat Sathyapalan ${ }^{3}$, Amirhossein Sahebkar $^{4,5,6^{*}}$

${ }^{1}$ Research Center of Physiology, Semnan University of Medical Sciences, Semnan, Iran

${ }^{2}$ Chronic Kidney Disease Research Center, Shahid Beheshti University of Medical Sciences, Tehran, Iran

${ }^{3}$ Academic Diabetes, Endocrinology and Metabolism, Hull York Medical School, University of Hull

${ }^{4}$ Halal Research Center of IRI, FDA, Tehran, Iran

${ }^{5}$ Biotechnology Research Center, Pharmaceutical Technology Institute, Mashhad University of Medical Sciences, Mashhad, Iran

${ }^{6}$ Neurogenic Inflammation Research Center, Mashhad University of Medical Sciences, Mashhad,

\section{*Corresponding Author}

Amirhossein Sahebkar, Biotechnology Research Center, Pharmaceutical Technology Institute, Mashhad University of Medical Sciences, Mashhad 9177948564, Iran. Tel: +985138002299; Fax: +985138002287; E-mail: sahebkara@mums.ac.ir; amir_saheb2000@yahoo.com

Habib Yaribeygi, Research Center of Physiology, Semnan University of Medical Sciences, Semnan, Iran, Tel: +989355644190

Running Title: Incretin based therapies and inflammation

(C)2019, Elsevier. This manuscript version is made available under the CC-BY-NCND 4.0 license http://creativecommons.org/licenses/by-nc-nd/4.0/ 


\begin{abstract}
GLP-1 receptor agonists (GLP-1RA) and dipeptidyl peptidase 4 inhibitors (DPP-4i) are two classes of antidiabetic agents used in the management of diabetes based on incretin hormones. There is emerging evidence that they have anti-inflammatory effects. Since most long-term complications of diabetes have a background of chronic inflammation, these agents may be beneficial against diabetic complications not only due to their hypoglycemic potentials but also via their anti-inflammatory effects. However, the exact molecular mechanisms by which GLP1RAs and DPP-4i exert their anti-inflammatory effects are not clearly understood. In this review, we discuss the potential molecular pathways by which these incretin-based therapies exert their anti-inflammatory effects.
\end{abstract}

Keywords: GLP-1 Receptor Agonist, DPP-4 Inhibitor, Diabetes Mellitus, Diabetic Complication, Inflammation. 


\section{Introduction}

The global prevalence of diabetes mellitus (DM) is rising rapidly (1). DM is a chronic disorder that carries considerable morbidity and mortality $(2,3)$. Chronic hyperglycemia due to DM triggers a cascade of pathophysiologic pathways such as oxidative stress, inflammation, apoptosis, necrosis, fibrosis, polyol pathway and hexosamine pathways leading to various diabetic complications $(2,4)$. Many classes of anti-diabetic medications have been developed for normalizing glycemia and prevent various complications of DM (5-7). The exact pathophysiology behind developing diabetes complications is not fully elucidated yet $(2,8)$, however, the inflammatory response has a considerable role in this (9-11).

Glucagon-like peptide-1 (GLP-1) is a small peptide belonging to the family of incretin hormones predominantly secreted by the intestinal L-cells (as well as by neurons) which reduces blood glucose by stimulation of insulin release and inhibition of glucagon secretion (12). Based on the potent hypoglycemic effects of this peptide, two classes of antidiabetic medications have been developed. They are an agonist of GLP-1 receptors (GLP-1 receptor agonists) and inhibitors of enzyme metabolizing GLP-1 (as dipeptidyl peptidase-4 inhibitors or DPP-4i) (13-15). These antidiabetic medications have a lower risk of hypoglycemia and thereby, safely normalize the hyperglycemia to physiologic levels (16). Beyond their hypoglycemia effects, some evidence indicates that they have anti-inflammatory potentials and potentially have a protective effect against various diabetic complications by lowering the inflammatory response $(17,18)$. If so, they can be considered as therapeutic agents for the management of inflammation-dependent diabetic complications (18). In this study, we discuss the anti-inflammatory effects of GLP-1RA and DPP-4i which could potentially protect various tissues against diabetic complications.

\section{GLP-1 RA and DPP-4i}


GLP-1RA is a class of antidiabetic agents that are approved by the FDA for the management of patients with diabetes (19). These medications reduce blood glucose in patients with diabetes by mimicking the effect of incretin hormones. Incretin hormones belong to a family of metabolic peptides including GLP-1 and GIP (gastric inhibitory peptide), which reduces postprandial glycemia by inhibition of glucagon secretion and stimulation of insulin release in a blood glucose-dependent manner (20-23). They can have additional physiologic effects such as delaying the gastric emptying, decreasing nutrient absorption, appetite suppression, improvement of lipid metabolism, inhibition of pancreatic $\beta$-cell apoptosis and induction of beta-cell neogenesis $(24,25,22)$. GLP-1 specific receptors are predominantly located in the pancreatic $\beta$-cells and are of G-protein coupled receptors that increase cAMP (cyclic adenosine monophosphate) production resulting in cellular events leading to insulin secretion from the pancreatic $\beta$-cells $(21,26)$. DPP-4i are a class of antidiabetic medications that produce their hypoglycemic effects through GLP-1 by increasing its active circulating levels by inhibiting DPP-4, which is an enzyme that inactivates GLP-1 $(27,28)$. Therefore, the DPP-4i have similar but less potent hypoglycemic effects compared to GLP-1RA, although they are differences in their effects on body weight and risk of some adverse effects (28).

\begin{tabular}{lccc}
\hline Classes & Approved Forms & Mechanisms of Action & Ref. \\
\hline \multirow{2}{*}{ GLP-1ra } & $\begin{array}{c}\text { Exenatide (Exendin-4), Liraglutide, } \\
\text { Semaglutide, Dulaglutide, } \\
\text { Lixisenatide, Albiglutide }\end{array}$ & $\begin{array}{c}\text { Mimic glucose-lowering effects of the } \\
\text { incretin-based hormones via glucagon } \\
\text { suppression, insulin release, appetite } \\
\text { inhibition, and slowing the gastric } \\
\text { emptying }\end{array}$ & $(20,23)$ \\
& Sitagliptin, Saxagliptin, Alogliptin, \\
LPP-4i & Linagliptin & Increase the circulatory levels of active & $(27,28)$ \\
\hline
\end{tabular}

Table 1; Two main classes of incretin-based diabetes medications

\section{Inflammatory hypothesis for the development of diabetic complications}


Various inflammatory pathways are activated in patients with diabetes $(9,29)$. There is growing evidence that there is activation of inflammatory pathways in the pathophysiology of various diabetes complications such as diabetic retinopathy, diabetic nephropathy, diabetic neuropathy and cardiovascular disease $(9,29,30,10)$. In addition, higher levels of inflammatory cytokines are typically found in the plasma of patients with diabetic complications $(29,31)$.

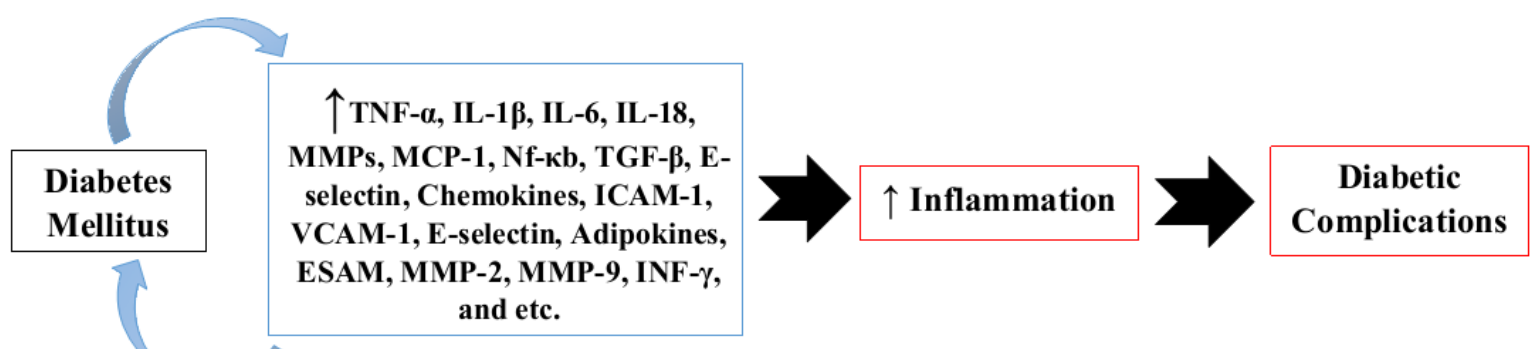

Figure 1; diabetes mellitus has bi-directional interaction with inflammatory mediators both of which intensify each other. This leads to inflammatory responses contributing to a higher risk for diabetic complications $(\mathrm{TNF}-\alpha=$ tumor necrosis factor-alpha, $\mathrm{IL}=$ interleukin, MMPs=matrix metalloproteinase, MCP-1=monocyte chemoattractant protein-1, nf-kb=nuclear factor kappa $\mathrm{b}$, TGF$\beta=$ transforming growth factor-beta, ICAM-1=intercellular adhesion molecule 1 , VCAM-1=vascular cell adhesion protein-1, ESAM=endothelial cell-selective adhesion molecule, MMP-2=matrix metalloproteinase-2, INF- $\gamma=$ interferon gamma)

Various inflammatory mediators such as TNF- $\alpha$ (tumor necrosis factor-alpha), IL (interleukin)1ß, IL-6, IL-18, MMPs (matrix metalloproteinase), MCP-1 (monocyte chemoattractant protein-1), nf-kb (nuclear factor kappa b), TGF- $\beta$ (transforming growth factor-beta), E-selectin, chemokines, different adhesion molecules as ICAM-1 (intercellular adhesion molecule 1) and VCAM-1 (vascular cell adhesion protein-1), TLRs (toll-like receptors), E-selectin, different adiponectin, endothelial cell-selective adhesion molecule (ESAM), MMP-2 (matrix metalloproteinase-2), MMP-9, and INF- $\gamma$ (interferon-gamma) are involved in various forms of diabetic complications (figure 1) (32-34). These mediators are highly expressed and secreted in diabetic milieu resulting in the development of, an "inflammation hypothesis" which emphasizes the crucial role of inflammatory responses in the pathophysiology of diabetic complications $(29,35)$.

Anti-inflammatory effects of GLP-1RA and DPP-4i 
These agents can exert anti-inflammatory effects via several molecular mechanisms and signaling pathways $(36,37)$. In the following paragraphs, we review the potential mechanisms by which GLP-1RA and DPP-4i exert these anti-inflammatory effects (table 2).

\section{Glucotoxicity}

GLP-1RA and DPP-4i are commonly used in the management of type 2 diabetes and have potent hypoglycemic effects and thereby attenuate glucotoxicity which is typically observed in a diabetic milieu $(38,39)$. GLP-1 has multiple physiologic effects that enable it to optimize glucose metabolism and reverse glucotoxicity (40-42). Glucotoxicity is associated with a higher incidence of inflammatory responses (43). Glucose-induced inflammation is well recognized in the hyperglycemic milieu and is reported in several studies (44-46). Therefore, one can speculate that by lowering the glucotoxicity via improvement of glucose homeostasis can be potentially translated into lower inflammatory responses (47).

Retnakaran and coworkers in 2014 reported that liraglutide (GLP-1RA) preserves beta-cell function by eliminating the glucotoxicity in patients with early T2DM (48). Also, Kong et al in 2014 reported that GLP-1 attenuated glucotoxicity by a mechanism dependent on RhoAROCK (Rho-associated kinase) signaling pathway in beta cells (40). Moreover, Tremblay et al in 2014 provided direct evidence in a clinical study demonstrating that sitagliptin markedly decreases inflammatory markers such as CRP (C-reactive protein), IL-6, IL-18 and E-selecting in plasma of patients with $\mathrm{T} 2 \mathrm{DM}$ (47). They demonstrated that the anti-inflammatory potential of sitagliptin is related to the improvement in glucose homeostasis (47). Although there is not enough direct evidence, intuitively, it is intuitive that eliminating the glucotoxicity via improvement in glucose homeostasis is one of the potential molecular mechanisms by which GLP-1RA and DPP-4i exert their anti-inflammatory effects.

\section{Recruitment of immune cells}


Recruitment of different types of immune cells such as T lymphocytes, NK (natural killer) cells, B-lymphocytes, leucocytes and dendritic cells into the inflamed tissues is a well-known event during inflammatory responses (49-51). Therefore, suppressing these phenomena and lowering the infiltration by immune cells is one of the main mechanisms for attenuating the inflammatory responses in various tissues $(50,52)$. There is some evidence implying that GLP1RA and DPP-4i can suppress or reduce the recruitment of immune cells $(36,37)$. Kodera and coworkers in 2011 found that exendin-4 ameliorates the inflammatory responses by preventing macrophage infiltration into the inflamed kidneys in diabetic rats (36). Also, Lee and colleagues in 2012 reported that GLP-1 reduced macrophage infiltration in adipocytes of obese mice (53). Moreover, Higashijima et al in 2015 demonstrated that DPP-4i (alogliptin) prevents the infiltration of CD68-macrophages into the kidneys in a non-diabetic milieu via GLP-1 specific signaling pathways (37). Wang et al in 2014 provided further evidence implying that exendin-4 reduced inflammatory responses by suppressing macrophage infiltration in inflamed liver tissues (54). It has suggested that GLP-1 exerts these anti-inflammatory effects via reducing the chemoattractant factors such as IFN- $\gamma$-induced STAT1 (signal transducer and activator of transcription-1) which is essential for T-cell recruitment $(55,18)$. Therefore, suppressing the leukocyte infiltration towards the inflamed tissue is another possible pathway by which GLP-1RA and DPP-4i inhibit inflammatory responses.

\section{Nf-kb signaling pathway and cytokines' expression}

$\mathrm{Nf}-\mathrm{kb}$ is a nuclear factor that is primarily recognized as a regulator of DNA expression but also plays essential roles in other cellular processes such as cell survival, response to external stimuli, synaptic plasticity and memory (56). It is a complex protein that regulates the transcription of various genes as well as inflammatory mediators and thereby, is responsible for immune responses and cytokine production in almost all types of human cells (57). Modulation of the nf-kb signaling pathway has been tried in different studies $(58,57)$. 
Emerging data demonstrate that GLP-1 can modulate nf- $\kappa b$ activities $(36,59-62)$. GLP-1RA and DPP-4i can potentially reduce the nf- $\mathrm{kb}$ activity in inflamed tissues that in turn downregulates the expression of pro-cytokines (59-62).

Lee and coworkers in 2012 found that GLP-1 reduced the nf-kb activation leading to lower cytokine production in the adipocytes of obese mice (53). Also, Kaidashev et al recently provided clinical evidence demonstrating that six weeks of liraglutide (GLP-1RA) therapy had an anti-inflammatory effect and reduced the expression of TNF- $\alpha$, IkB, TLR2, and TLR4 via nf-kb inhibition in patients with T2DM (62). Furthermore, Nader and coworkers in 2018 revealed that sitagliptin markedly reduces the nf-kb function in non-diabetic mice (61). ElSahar and coworkers in 2015 have shown that sitagliptin exerts its anti-inflammatory effects at least partly via $\mathrm{nf}-\mathrm{\kappa b}$ inhibition (60). This evidence strongly suggest that inhibition of nf-kb signaling pathways is another potential mechanism by which GLP-1 and DPP-4i provide antiinflammatory potentials in both diabetic and non-diabetic milieu.

However, these anti-diabetic medications can also suppress the expression of cytokines via nf$\kappa b$ independent pathways (63). Velmurugan and coworkers in 2012 reported that exendin-4 prevent cytokines' expression by a CREB (cAMP response element-binding protein) dependent mechanism in the pancreatic islets (63). Also, He et al in 2013 found that exendin-4 ameliorated the expression of inflammatory mediators by p38 MAPK (phospho-mitogen activated protein kinase) pathways in CD4+ $\mathrm{T}$ cells (64). Moreover, Que and coworkers suggested that liraglutide (GLP-1RA) down-regulated the cytokines through the PKA/CREB pathway in rats (65). This evidence strongly suggests that GLP-1 can prevent the expression of cytokines not only via nf- $\kappa b$ modulation but also through other molecular mechanisms such as CREB and p38 MAPK pathways (63-65).

\section{Oxidative stress}


Oxidative stress is a common event in the diabetic milieu (66). This deleterious state induces other pathophysiologic pathways such as apoptosis, fibrosis, autophagy and inflammation (67). It has well confirmed that oxidative stress has dual interaction with inflammatory responses which both intensify others $(67,68)$. Thus, improvement in redox state translated to a lower incidence of inflammation $(69,68)$. GLP-1RA and DPP-4i can improve oxidative stress via several pathways such as nuclear factor erythroid 2-related factor 2 (nrf2) pathway, potentiation of antioxidant defense system, down-regulation of pro-oxidant enzymes such as $\mathrm{NAD}(\mathrm{P}) \mathrm{H}$ oxidase thereby lowering the free radical generation and improvement in glucose metabolism leading to lesser amount of toxic by-products such as AGEs (advanced glycation end-products) (70-72).

There is strong evidence indicating that GLP-1RA and DPP-4i inhibit inflammatory responses through the re-adjustment of the redox state $(73,64,60,70,74-76,61)$. He et al in 2013 found that exendin- 4 ameliorates the inflammatory processes by lowering the oxidative stress in patients with diabetes (64). Also, Chen and coworkers in 2017 demonstrated that exendin-4 can reduce inflammation via its antioxidative potentials in diabetic rats (74). Moreover, Chang and colleagues in 2017 reported that exendin-4 attenuates AGEs-induced inflammatory responses by its antioxidative effects in the mesangial cells of diabetic rats (70). Moustafa et al in 2018 provided further evidence indicating that anti-inflammatory potentials of liraglutide are at least partially related to its antioxidative effects (76). This evidence strongly suggests that GLP-1RA and DPP-4i can attenuate the inflammatory events via normalizing the redox state and thereby reducing oxidative stress-induced inflammation $(73,60,75,61)$. 


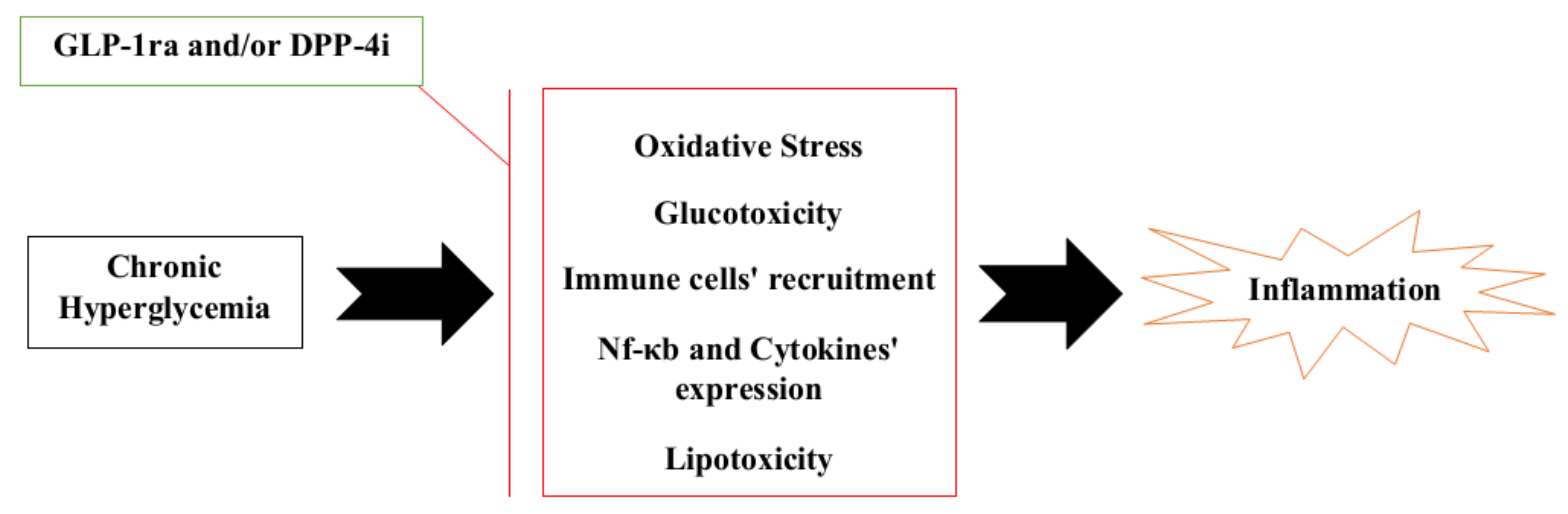

Figure 2; GLP-1RA and DPP-4i suppress molecular pathways involved in diabetesinduced inflammatory responses

\section{Lipotoxicity}

Lipotoxicity occurs when the excess byproducts of lipid metabolism accumulate in nonadipose tissues which in turn, trigger a cascade of deleterious pathways such as apoptosis and inflammation (77). Some studies emphasized the role of lipotoxicity as a missing link between diabetes and various inflammatory disorders $(77,78)$. They suggested that lipotoxicity is the main cause of inflammatory processes in a diabetic milieu $(79,78)$. Thus, improving the lipid metabolism and correcting the plasma lipid profile can prevent or suppress inflammatory responses $(80,79,81)$. There is some evidence confirming that GLP-1RA and DPP-4i have this effect $(73,82-84)$.

Huang et al in 2015 found that intra-islet GLP-1 secretion is a potent intrinsic defense against lipotoxicity (82). They also reported that liraglutide can prevent lipotoxicity and in turn, inhibits the inflammatory response (82). Pastel and coworkers in 2016 illustrated that exendin4 ameliorates the inflammatory processes via improvement in lipid metabolism in cultured human adipocytes (83). Similarly, Ferreira and coworkers in 2010 found that can reduce inflammatory events through correcting the lipid metabolism in diabetic animals (73). Yin et al in 2016 demonstrated that exendin-4 attenuates lipotoxicity by correcting cholesterol homeostasis in the glomerular cells of diabetic mice (84). They also found that this effect was 
accompanied by lowering the inflammatory responses in this tissue (84). More studies have reported the same results implying that GLP-1RA and DPP-4i prevent lipotoxicity in various tissues $(85,86)$. This evidence suggests that these classes of compounds can suppress inflammatory events through ameliorating lipotoxicity.

\begin{tabular}{|c|c|c|}
\hline Molecular Mechanisms & Effects of GLP-1ra and/or DPP-4i & Ref. \\
\hline Glucotoxicity & $\begin{array}{l}\text { Eliminate glucotoxicity leading to a lower } \\
\text { rate of glucose-induced inflammation }\end{array}$ & $\begin{array}{c}(40,41,48,47, \\
42-46)\end{array}$ \\
\hline $\begin{array}{l}\text { Immune cells' } \\
\text { recruitment }\end{array}$ & $\begin{array}{l}\text { Reduce leukocyte immigration toward the } \\
\text { inflamed tissue }\end{array}$ & $(36,37)$ \\
\hline $\begin{array}{l}\text { Nf-kb and } \\
\text { Cytokines' expression }\end{array}$ & $\begin{array}{l}\text { Directly down-regulate different } \\
\text { inflammatory cytokines in several ways } \\
\text { such as nf-kb inhibition }\end{array}$ & $\begin{array}{c}(59-62) \\
(55,63,64,83, \\
70,74,76,87- \\
89,62,65)\end{array}$ \\
\hline Lipotoxicity & $\begin{array}{l}\text { Correct lipid homeostasis leading to a lower } \\
\text { rate of lipotoxicity-induced inflammation }\end{array}$ & $(73,82,83)$ \\
\hline Oxidative stress & $\begin{array}{l}\text { Improve redox state toward physiologic } \\
\text { state leading to lower oxidative stress- } \\
\text { induced inflammation }\end{array}$ & $\begin{array}{l}(73,64,60,70 \\
\quad 74-76,61)\end{array}$ \\
\hline
\end{tabular}

Table 2; Possible molecular mechanisms by which GLP-1RA and DPP-4i exert their anti-inflammatory effects 


\begin{tabular}{|c|l|l|c|}
\hline Treatment & Population of study & Mechanism of reducing inflammation & Ref. \\
\hline Exendin-4 & Patients with T2DM & Normalize redox state & $(64)$ \\
\hline Liraglutide & Patients with T2DM & Reduce the glucotoxicity & $(48)$ \\
\hline Liraglutide & Patients with T2DM & $\begin{array}{l}\text { Decline nf-kb dependent cytokines' } \\
\text { expression }\end{array}$ & $(62)$ \\
\hline Sitagliptin & Patients with T2DM & Reduce the glucotoxicity & $(47)$ \\
\hline Sitagliptin & Patients with T2DM & $\begin{array}{l}\text { Reduce the glucotoxicity, down-regulate } \\
\text { inflammatory mediators }\end{array}$ & $(90)$ \\
\hline Sitagliptin & $\begin{array}{c}\text { Patients with T2DM } \\
\text { Improve glucose homeostasis and thereby } \\
\text { reduce glucotoxicity }\end{array}$ & $(47)$ \\
\hline Sitagliptin & $\begin{array}{c}\text { Patients with T2DM } \\
\text { and cardiovascular } \\
\text { diseases }\end{array}$ & $\begin{array}{l}\text { Normalize glucose homeostasis and reduce } \\
\text { inflammatory cytokines }\end{array}$ & $(91)$ \\
\hline
\end{tabular}

Table 3; Clinical studies on anti-inflammatory potentials of GLP-1RA and DPP-4i

\section{Conclusion}

GLP-1RA and DPP-4i are novel classes of anti-diabetic medications, in addition to their hypoglycemic effects can provide anti-inflammatory effects on various tissues. They can potentially prevent inflammatory events via at least five molecular mechanisms such as improvement in glucose homeostasis and lowering the glucotoxicity, normalizing the redox state and reducing the oxidative stress-induced inflammation, suppressing the leukocyte recruitment towards the inflamed tissue, reducing the lipotoxicity and down-regulation of various inflammatory mediators. These agents may be considered as potential therapeutic agents against various inflammatory disorders as well as diabetic complications.

\section{Acknowledgment}

The authors are thankful to the "Research center of physiology, Semnan University of medical sciences (Semnan, Iran)" for providing technical supports. 


\section{Conflict of Interests}

The authors declare that have no conflict of interest in this study. 


\section{References}

[1] E. J. Mayer-Davis, J. M. Lawrence, D. Dabelea, J. Divers, S. Isom, L. Dolan, G. Imperatore, B. Linder, S. Marcovina, D. J. Pettitt (2017) Incidence trends of type 1 and type 2 diabetes among youths, 2002-2012. New England Journal of Medicine 376:1419-1429.

[2] J. M. Forbes, M. E. Cooper (2013) Mechanisms of diabetic complications. Physiological reviews 93:137-188.

[3] M. A. Reddy, E. Zhang, R. Natarajan (2015) Epigenetic mechanisms in diabetic complications and metabolic memory. Diabetologia 58:443-455.

[4] C. M. O. Volpe, P. H. Villar-Delfino, P. M. F. dos Anjos, J. A. Nogueira-Machado (2018) Cellular death, reactive oxygen species (ROS) and diabetic complications. Cell death \& disease 9:119.

[5] A. D. Association (2014) Diagnosis and classification of diabetes mellitus. Diabetes care 37:S81S90.

[6] H. Yaribeygi, S. L. Atkin, A. Sahebkar (2019) Natural compounds with DPP-4 inhibitory effects: Implications for the treatment of diabetes. Journal of cellular biochemistry 120:10909-

10913.

[7] H. Yaribeygi, F. Lhaf, T. Sathyapalan, A. Sahebkar (2019) Effects of novel antidiabetes agents on apoptotic processes in diabetes and malignancy: Implications for lowering tissue damage.

Life sciences.

[8] K. Papatheodorou, N. Papanas, M. Banach, D. Papazoglou, M. Edmonds (2016) Complications of diabetes 2016. Journal of diabetes research 2016.

[9] M. D. Williams, J. L. Nadler (2007) Inflammatory mechanisms of diabetic complications. Current diabetes reports 7:242-248.

[10] H. Yaribeygi, S. L. Atkin, A. Sahebkar (2019) Interleukin-18 and diabetic nephropathy: A review. Journal of cellular physiology 234:5674-5682.

[11] H. Yaribeygi, N. Katsiki, A. E. Butler, A. Sahebkar (2019) Effects of antidiabetic drugs on NLRP3 inflammasome activity, with a focus on diabetic kidneys. Drug discovery today 24:256-262.

[12] J. J. Holst (2007) The physiology of glucagon-like peptide 1. Physiological reviews 87:1409-1439. [13] H. Bihan, W. L. Ng, D. J. Magliano, J. E. Shaw (2016) Predictors of efficacy of GLP-1 agonists and DPP-4 inhibitors: a systematic review. Diabetes research and clinical practice 121:27-34.

[14] V. A. Gault, C. Hölscher (2018) GLP-1 receptor agonists show neuroprotective effects in animal models of diabetes. Peptides 100:101-107.

[15] H. Yaribeygi, T. Sathyapalan, A. Sahebkar (2019) Molecular mechanisms by which GLP-1 RA and DPP-4i induce insulin sensitivity. Life sciences 116776.

[16] B. Zinman, J. Gerich, J. B. Buse, A. Lewin, S. Schwartz, P. Raskin, P. M. Hale, M. Zdravkovic, L. Blonde (2009) Efficacy and safety of the human GLP-1 analog liraglutide in combination with metformin and TZD in patients with type 2 diabetes mellitus (LEAD-4 Met+ TZD). Diabetes care.

[17] N. M. Krasner, Y. Ido, N. B. Ruderman, J. M. Cacicedo (2014) Glucagon-like peptide-1 (GLP-1) analog liraglutide inhibits endothelial cell inflammation through a calcium and AMPK dependent mechanism. Plos one 9:e97554.

[18] Y.-S. Lee, H.-S. Jun (2016) Anti-inflammatory effects of GLP-1-based therapies beyond glucose control. Mediators of inflammation 2016.

[19] H. Yaribeygi, S. L. Atkin, M. Pirro, A. Sahebkar (2018) A review of the anti-inflammatory properties of antidiabetic agents providing protective effects against vascular complications in diabetes. Journal of cellular physiology.

[20] D. J. Drucker, M. A. Nauck (2006) The incretin system: glucagon-like peptide-1 receptor agonists and dipeptidyl peptidase-4 inhibitors in type 2 diabetes. The Lancet 368:1696-1705.

[21] L. L. Baggio, D. J. Drucker (2007) Biology of incretins: GLP-1 and GIP. Gastroenterology 132:21312157.

[22] J. J. Meier (2012) GLP-1 receptor agonists for individualized treatment of type 2 diabetes mellitus. Nature Reviews Endocrinology 8:728. 
[23] M. Islam (2016) Insulinotropic Effect of Herbal Drugs for Management of Diabetes Mellitus: A Congregational Approach. Biosens J 5:2.

[24] X. Ding, N. K. Saxena, S. Lin, N. Gupta, F. A. Anania (2006) Exendin-4, a glucagon-like protein-1 (GLP-1) receptor agonist, reverses hepatic steatosis in ob/ob mice. Hepatology 43:173-181.

[25] K. A. Scott, T. H. Moran (2007) The GLP-1 agonist exendin-4 reduces food intake in nonhuman primates through changes in meal size. American Journal of Physiology-Regulatory, Integrative and Comparative Physiology 293:R983-R987.

[26] D. Wootten, J. Simms, C. Koole, O. L. Woodman, R. J. Summers, A. Christopoulos, P. M. Sexton (2011) Modulation of the glucagon-like peptide-1 receptor signaling by naturally occurring and synthetic flavonoids. Journal of Pharmacology and Experimental Therapeutics 336:540550.

[27] B. Ahren (2007) DPP-4 inhibitors. Best Practice \& Research Clinical Endocrinology \& Metabolism 21:517-533.

[28] A. D. Association (2018) 2. Classification and diagnosis of diabetes: standards of medical care in diabetes-2018. Diabetes Care 41:S13-S27.

[29] G. L. King (2008) The role of inflammatory cytokines in diabetes and its complications. Journal of periodontology 79:1527-1534.

[30] M. Capitão, R. Soares (2016) Angiogenesis and inflammation crosstalk in diabetic retinopathy. Journal of cellular biochemistry 117:2443-2453.

[31] M. S. Ellulu, I. Patimah, H. Khaza'ai, A. Rahmat, Y. Abed (2017) Obesity and inflammation: the linking mechanism and the complications. Archives of medical science: AMS 13:851.

[32] J. F. Navarro-González, C. Mora-Fernández, M. M. De Fuentes, J. García-Pérez (2011) Inflammatory molecules and pathways in the pathogenesis of diabetic nephropathy. Nature Reviews Nephrology 7:327.

[33] J. Donate-Correa, E. Martín-Núñez, M. Muros-de-Fuentes, C. Mora-Fernández, J. F. NavarroGonzález (2015) Inflammatory cytokines in diabetic nephropathy. Journal of diabetes research 2015.

[34] G. Garibotto, A. Carta, D. Picciotto, F. Viazzi, D. Verzola (2017) Toll-like receptor-4 signaling mediates inflammation and tissue injury in diabetic nephropathy. Journal of nephrology

30:719-727.

[35] C. Garcia, B. Feve, P. Ferre, S. Halimi, H. Baizri, L. Bordier, G. Guiu, O. Dupuy, B. Bauduceau, H. Mayaudon (2010) Diabetes and inflammation: fundamental aspects and clinical implications.

Diabetes \& metabolism 36:327-338.

[36] R. Kodera, K. Shikata, H. Kataoka, T. Takatsuka, S. Miyamoto, M. Sasaki, N. Kajitani, S. Nishishita, K. Sarai, D. Hirota (2011) Glucagon-like peptide-1 receptor agonist ameliorates renal injury through its anti-inflammatory action without lowering blood glucose level in a rat model of type 1 diabetes. Diabetologia 54:965-978.

[37] Y. Higashijima, T. Tanaka, J. Yamaguchi, S. Tanaka, M. Nangaku (2015) Anti-inflammatory role of DPP-4 inhibitors in a nondiabetic model of glomerular injury. American Journal of Physiology-Renal Physiology 308:F878-F887.

[38] S. Del Prato (2009) Role of glucotoxicity and lipotoxicity in the pathophysiology of Type 2 diabetes mellitus and emerging treatment strategies. Diabetic Medicine 26:1185-1192.

[39] G. Luo, X. Kong, L. Lu, X. Xu, H. Wang, X. Ma (2013) Glucagon-like peptide 1 potentiates glucotoxicity-diminished insulin secretion via stimulation of CAMP-PKA signaling in INS-1E cells and mouse islets. The international journal of biochemistry \& cell biology 45:483-490. [40] X. Kong, D. Yan, J. Sun, X. Wu, H. Mulder, X. Hua, X. Ma (2014) Glucagon-like peptide 1 stimulates insulin secretion via inhibiting RhoA/ROCK signaling and disassembling glucotoxicity-induced stress fibers. Endocrinology 155:4676-4685.

[41] P. Nadkarni, O. G. Chepurny, G. G. Holz in: Progress in molecular biology and translational science, (2014), Elsevier, pp 23-65. 
[42] I. Tsavdaridis, D. Papadimitriou, D. Karanikola, K. Kalousis, A. Katsouda, M. MironidouTzouveleki (2015) Sitagliptin reduces urinary microalbumin in experimental model of diabetic nephropathy. Hellenic journal of nuclear medicine 18:154-154.

[43] S. Berdja, L. Smail, B. Saka, S. Neggazi, E.-m. Haffaf, Y. Benazzoug, G. Kacimi, L. Boudarene, S. Aouichat Bouguerra (2016) Glucotoxicity induced oxidative stress and inflammation in vivo and in vitro in Psammomys obesus: involvement of aqueous extract of Brassica rapa rapifera. Evidence-Based Complementary and Alternative Medicine 2016.

[44] H. Chen, X. Yang, K. Lu, C. Lu, Y. Zhao, S. Zheng, J. Li, Z. Huang, Y. Huang, Y. Zhang (2017) Inhibition of high glucose-induced inflammation and fibrosis by a novel curcumin derivative prevents renal and heart injury in diabetic mice. Toxicology letters 278:48-58.

[45] F. Chen, X. Zhu, Z. Sun, Y. Ma (2018) Astilbin inhibits high glucose-induced inflammation and extracellular matrix accumulation by suppressing the TLR4/MyD88/NF-KB pathway in rat glomerular mesangial cells. Frontiers in pharmacology 9.

[46] K.-C. Lee, J. S. Lee, S. Jo, D. Kim, S. Y. Han (2018) High glucose induces inflammatory reactions and changes in histone-modifying enzymes in rat mesangial cells. Biomedical Research 29:1103-1109.

[47] A. J. Tremblay, B. Lamarche, C. F. Deacon, S. J. Weisnagel, P. Couture (2014) Effects of sitagliptin therapy on markers of low-grade inflammation and cell adhesion molecules in patients with type 2 diabetes. Metabolism 63:1141-1148.

[48] R. Retnakaran, C. K. Kramer, H. Choi, B. Swaminathan, B. Zinman (2014) Liraglutide and the preservation of pancreatic $\beta$-cell function in early type 2 diabetes: the LIBRA trial. Diabetes care 37:3270-3278.

[49] J.-Q. Gao, N. Okada, T. Mayumi, S. Nakagawa (2008) Immune cell recruitment and cell-based system for cancer therapy. Pharmaceutical research 25:752-768.

[50] D. Vestweber (2015) How leukocytes cross the vascular endothelium. Nature Reviews Immunology 15:692.

[51] M. Schnoor, P. Alcaide, M.-B. Voisin, J. D. van Buul (2016) Recruitment of immune cells into inflamed tissues: consequences for endothelial barrier integrity and tissue functionality.

Mediators of inflammation 2016.

[52] D. R. Lacerda, M. M. Moraes, A. Nunes-Silva, K. A. Costa, D. F. Rodrigues, J. L. Sabino, L. M. d. S. Cordeiro, V. Pinho, M. M. Teixeira, S. P. Wanner (2018) Aerobic training reduces immune cell recruitment and cytokine levels in adipose tissue in obese mice. Applied Physiology, Nutrition, and Metabolism 44:512-520.

[53] Y.-S. Lee, M.-S. Park, J.-S. Choung, S.-S. Kim, H.-H. Oh, C.-S. Choi, S.-Y. Ha, Y. Kang, Y. Kim, H.-S. Jun (2012) Glucagon-like peptide-1 inhibits adipose tissue macrophage infiltration and inflammation in an obese mouse model of diabetes. Diabetologia 55:2456-2468.

[54] Y. Wang, E. Parlevliet, J. Geerling, S. Van Der Tuin, H. Zhang, V. Bieghs, A. Jawad, R. ShiriSverdlov, I. Bot, S. De Jager (2014) Exendin-4 decreases liver inflammation and atherosclerosis development simultaneously by reducing macrophage infiltration. British journal of pharmacology 171:723-734.

[55] U. Pugazhenthi, K. Velmurugan, A. Tran, G. Mahaffey, S. Pugazhenthi (2010) Anti-inflammatory action of exendin- 4 in human islets is enhanced by phosphodiesterase inhibitors: potential therapeutic benefits in diabetic patients. Diabetologia 53:2357-2368.

[56] P. P. Tak, G. S. Firestein (2001) NF-kB: a key role in inflammatory diseases. The Journal of clinical investigation 107:7-11.

[57] T. D. Gilmore (2006) Introduction to NF-KB: players, pathways, perspectives. Oncogene 25:6680.

[58] K. M. Lee, B. S. Kang, H. L. Lee, S. J. Son, S. H. Hwang, D. S. Kim, J. S. Park, H. J. Cho (2004) Spinal NF-kB activation induces COX-2 upregulation and contributes to inflammatory pain hypersensitivity. European Journal of Neuroscience 19:3375-3381. 
[59] A. Makdissi, H. Ghanim, M. Vora, K. Green, S. Abuaysheh, A. Chaudhuri, S. Dhindsa, P. Dandona (2012) Sitagliptin exerts an antinflammatory action. The Journal of Clinical Endocrinology \& Metabolism 97:3333-3341.

[60] A. E. El-Sahar, M. M. Safar, H. F. Zaki, A. S. Attia, A. A. Ain-Shoka (2015) Sitagliptin attenuates transient cerebral ischemia/reperfusion injury in diabetic rats: Implication of the oxidativeinflammatory-apoptotic pathway. Life sciences 126:81-86.

[61] M. A. Nader, H. Ateyya, M. El-Shafey, N. A. El-Sherbeeny (2018) Sitagliptin enhances the neuroprotective effect of pregabalin against pentylenetetrazole-induced acute epileptogenesis in mice: implication of oxidative, inflammatory, apoptotic and autophagy pathways. Neurochemistry international 115:11-23.

[62] I. Kaidashev, L. G. Savchenko, N. I. Digtiar, L. G. Selikhova, E. I. Kaidasheva, O. A. Shlykova, L. E. Vesnina (2019) Liraglutide exerts an anti-inflammatory action in obese patients with type 2 diabetes. Romanian Journal of Internal Medicine 1.

[63] K. Velmurugan, A. Balamurugan, G. Loganathan, A. Ahmad, B. J. Hering, S. Pugazhenthi (2012) Antiapoptotic actions of exendin-4 against hypoxia and cytokines are augmented by CREB. Endocrinology 153:1116-1128.

[64] L. He, C. K. Wong, K. K. Cheung, H. C. Yau, A. Fu, H. I. Zhao, K. M. Leung, A. P. Kong, G. W. Wong, P. K. Chan (2013) Anti-inflammatory effects of exendin-4, a glucagon-like peptide-1 analog, on human peripheral lymphocytes in patients with type 2 diabetes. Journal of diabetes investigation 4:382-392.

[65] Q. Que, X. Guo, L. Zhan, S. Chen, Z. Zhang, X. Ni, B. Ye, S. Wan (2019) The GLP-1 agonist, liraglutide, ameliorates inflammation through the activation of the PKA/CREB pathway in a rat model of knee osteoarthritis. Journal of Inflammation 16:13.

[66] J. L. Rains, S. K. Jain (2011) Oxidative stress, insulin signaling, and diabetes. Free Radical Biology and Medicine 50:567-575.

[67] P. Dandona, A. Aljada, A. Bandyopadhyay (2004) Inflammation: the link between insulin resistance, obesity and diabetes. Trends in immunology 25:4-7.

[68] B. L. Tan, M. E. Norhaizan, H. S. Winnie-Pui-Pui Liew (2018) Antioxidant and oxidative stress: A mutual interplay in age-related diseases. Frontiers in pharmacology 9.

[69] R. Fischer, O. Maier (2015) Interrelation of oxidative stress and inflammation in neurodegenerative disease: role of TNF. Oxidative medicine and cellular longevity 2015.

[70] J.-T. Chang, Y.-J. Liang, C.-Y. Hsu, C.-Y. Chen, P.-J. Chen, Y.-F. Yang, Y.-L. Chen, D. Pei, J.-B. Chang, J.-G. Leu (2017) Glucagon-like peptide receptor agonists attenuate advanced glycation end products-induced inflammation in rat mesangial cells. BMC Pharmacology and Toxicology 18:67.

[71] Y. Oh, H.-S. Jun (2017) Effects of glucagon-like peptide-1 on oxidative stress and Nrf2 signaling. International journal of molecular sciences 19:26.

[72] Ł. Bułdak, G. Machnik, E. Skudrzyk, A. Bołdys, B. Okopień (2019) The impact of exenatide (a GLP-1 agonist) on markers of inflammation and oxidative stress in normal human astrocytes subjected to various glycemic conditions. Experimental and therapeutic medicine 17:28612869.

[73] L. Ferreira, E. Teixeira-de-Lemos, F. Pinto, B. Parada, C. Mega, H. Vala, R. Pinto, P. Garrido, J. Sereno, R. Fernandes (2010) Effects of sitagliptin treatment on dysmetabolism, inflammation, and oxidative stress in an animal model of type 2 diabetes (ZDF rat).

Mediators of inflammation 2010.

[74] Y.-C. Chen, C.-C. Ho, C.-H. Yi, X.-Z. Liu, T.-T. Cheng, C.-F. Lam (2017) Exendin-4, a glucagon-like peptide-1 analogue accelerates healing of chronic gastric ulcer in diabetic rats. PloS one 12:e0187434.

[75] C. H. Jo, S. Kim, J.-S. Park, G.-H. Kim (2018) Anti-inflammatory action of sitagliptin and linagliptin in doxorubicin nephropathy. Kidney and Blood Pressure Research 43:987-999. 
[76] P. E. Moustafa, N. F. Abdelkader, S. A. El Awdan, O. A. El-Shabrawy, H. F. Zaki (2018) Liraglutide ameliorated peripheral neuropathy in diabetic rats: involvement of oxidative stress, inflammation and extracellular matrix remodeling. Journal of neurochemistry 146:173-185.

[77] R. A. DeFronzo (2010) Insulin resistance, lipotoxicity, type 2 diabetes and atherosclerosis: the missing links. The Claude Bernard Lecture 2009. Diabetologia 53:1270-1287.

[78] S. Savary, D. Trompier, P. Andréoletti, F. Le Borgne, J. Demarquoy, G. Lizard (2012) Fatty acidsinduced lipotoxicity and inflammation. Current drug metabolism 13:1358-1370.

[79] K. Cusi (2010) The role of adipose tissue and lipotoxicity in the pathogenesis of type 2 diabetes. Current diabetes reports 10:306-315.

[80] R. A. Defronzo (2004) Dysfunctional fat cells, lipotoxicity and type 2 diabetes. International journal of clinical practice 58:9-21.

[81] N. Mendez-Sanchez, V. Cruz-Ramon, O. Ramirez-Perez, J. Hwang, B. Barranco-Fragoso, J. Cordova-Gallardo (2018) New aspects of lipotoxicity in nonalcoholic steatohepatitis. International journal of molecular sciences 19:2034.

[82] C. Huang, L. Yuan, S. Cao (2015) Endogenous GLP-1 as a key self-defense molecule against lipotoxicity in pancreatic islets. International journal of molecular medicine 36:173-185.

[83] E. Pastel, S. Joshi, B. Knight, N. Liversedge, R. Ward, K. Kos (2016) Effects of Exendin-4 on human adipose tissue inflammation and ECM remodelling. Nutrition \& diabetes 6:e235.

[84] Q.-h. Yin, R. Zhang, L. Li, Y.-t. Wang, J.-p. Liu, J. Zhang, L. Bai, J.-q. Cheng, P. Fu, F. Liu (2016)

Exendin-4 ameliorates lipotoxicity-induced glomerular endothelial cell injury by improving

ABC transporter A1-mediated cholesterol efflux in diabetic apoE knockout mice. Journal of Biological Chemistry 291:26487-26501.

[85] Q. Wei, Y. Q. Sun, J. Zhang (2012) Exendin-4, a glucagon-like peptide-1 receptor agonist, inhibits cell apoptosis induced by lipotoxicity in pancreatic $\beta$-cell line. Peptides 37:18-24.

[86] M. J. Armstrong, D. Hull, K. Guo, D. Barton, J. M. Hazlehurst, L. L. Gathercole, M. Nasiri, J. Yu, S. C. Gough, P. N. Newsome (2016) Glucagon-like peptide 1 decreases lipotoxicity in nonalcoholic steatohepatitis. Journal of hepatology 64:399-408.

[87] G. Rakipovski, B. Rolin, J. Nøhr, I. Klewe, K. S. Frederiksen, R. Augustin, J. Hecksher-Sørensen, C. Ingvorsen, J. Polex-Wolf, L. B. Knudsen (2018) The GLP-1 analogs liraglutide and semaglutide reduce atherosclerosis in ApoE-/- and LDLr-/- mice by a mechanism that includes inflammatory pathways. JACC: Basic to Translational Science 3:844-857.

[88] W.-R. Shen, K. Kimura, M. Ishida, H. Sugisawa, A. Kishikawa, K. Shima, S. Ogawa, J. Qi, H. Kitaura (2018) The glucagon-like peptide-1 receptor agonist exendin-4 inhibits lipopolysaccharideinduced osteoclast formation and bone resorption via inhibition of TNF- $\alpha$ expression in macrophages. Journal of immunology research 2018.

[89] S. Borzouei, V. Sheikh, M. Ghasemi, A. Zamani, Z. Telikani, Z. Zareighane, I. Salehi, A. Mozayanimonfared, M. A. Amirzargar, M. Alahgholi-Hajibehzad (2019) Anti-inflammatory effect of combined sitagliptin and vitamin D3 on cytokines profile in patients with type 2 diabetes mellitus. Journal of Interferon \& Cytokine Research 39:293-301.

[90] B. Charbonnel, A. Karasik, J. Liu, M. Wu, G. Meininger (2006) Efficacy and safety of the dipeptidyl peptidase-4 inhibitor sitagliptin added to ongoing metformin therapy in patients with type 2 diabetes inadequately controlled with metformin alone. Diabetes care 29:26382643.

[91] J. Matsubara, S. Sugiyama, E. Akiyama, S. Iwashita, H. Kurokawa, K. Ohba, H. Maeda, K. Fujisue, E. Yamamoto, K. Kaikita (2013) Dipeptidyl peptidase-4 inhibitor, sitagliptin, improves endothelial dysfunction in association with its anti-inflammatory effects in patients with coronary artery disease and uncontrolled diabetes. Circulation Journal 77:1337-1344. 